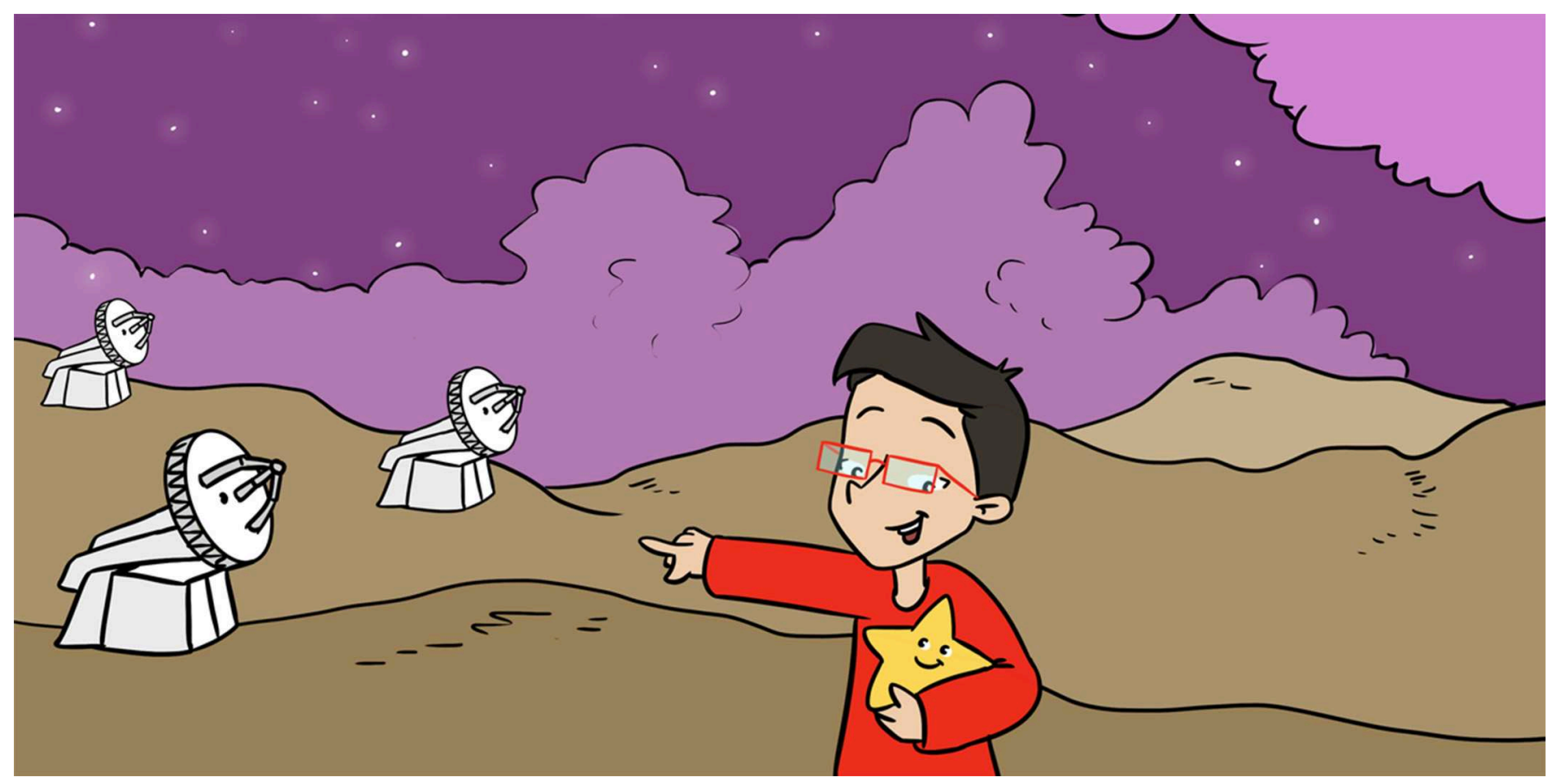

\title{
HOW DO STARS FORM?
}

\section{Majken Brahe Ellegaard Christensen *}

Astronomicca, Odense, Denmark

\section{YOUNG REVIEWER}

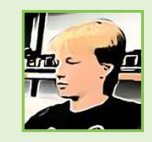

LUKE

AGE: 15
In this article we explain the process of star formation for regular Sun-like stars. Stars form from an accumulation of gas and dust, which collapses due to gravity and starts to form stars. The process of star formation takes around a million years from the time the initial gas cloud starts to collapse until the star is created and shines like the Sun. The leftover material from the star's birth is used to create planets and other objects that orbit the central star. Observing star formation is difficult, because the dust is not transparent to visible light. It is, however, possible to observe these dark stellar nurseries using radio waves, because radio waves travel freely down to us and our radio telescopes.

Stars, like our own Sun, have not always been around. Stars are born and die over millions or even billions of years. Stars form when regions of dust and gas in the galaxy collapse due to gravity. Without this dust and gas, stars would not form. 
INTERSTELLAR

MEDIUM (ISM)

The entire space inside galaxies where there are no stars, but instead a lot of gas and dust.

\section{MOLECULAR}

CLOUD

A large space cloud filled with gas and dust Molecular clouds are found inside the interstellar medium.

\section{AU}

1 Astronomical Unit, which is the distance from the Earth to the Sun.

\section{ABSOLUTE ZERO}

The lowest possible temperature, which is also called $0^{\circ} \mathrm{K}$

\section{THE DUSTY NUSERIES OF STARS}

A galaxy contains not only billions of stars, but also large amounts of gas and dust. These regions of gas and dust in the galaxy lie in the space between the stars. If the galaxy were a street, the houses would be stars and the regions of gas and dust would be the gardens in between the houses. The space between the stars in a galaxy is called the interstellar medium, because it is the medium, or substance, that makes up the space between stellar objects.

The regions of gas and dust are called molecular clouds, because of their content. Molecular clouds are made of a mix of atoms, molecules, and dust. Atoms are the small building blocks of all the stuff around us. Molecules consist of two or more atoms joined together. The molecules present in molecular clouds are typically molecular hydrogen, $\mathrm{H}_{2}$, but can also be more complex molecules, such as methanol, which consists of six atoms, or water, which consists of three atoms. Dust grains are even larger clumps of matter and they can be up to a few millimeters in size, which is huge compared with atoms or molecules.

Molecular clouds in the interstellar medium are large. In fact, a single molecular cloud can be hundreds of thousands of times heavier than the Sun. Their volumes also vary: a molecular cloud can be the same size as, or many times bigger than, our entire solar system. These enormous molecular clouds undergo turbulent motion. This means that the gas and dust within the clouds do not stay in the same place as time passes. These substances move around in all directions, like children running around in a school yard. This turbulent motion of the gas and dust distributes the atoms and molecules unevenly, so that some regions of the molecular cloud will have more matter in them than other regions Figure $1 \mathrm{~A}$. If the gas and dust pile up to a very high level in a certain region, that region starts to collapse due to the pull from its own gravity. The region is smaller than the molecular cloud and lives inside the molecular cloud. The region is "only" a few hundred Astronomical Units (AUs), which is a few hundred times the distance from the Earth to the Sun.

\section{THE COLLAPSING MOLECULAR CLOUD}

A molecular cloud is very cold, only a few degrees above absolute zero, which is the lowest temperature possible (also called $0^{\circ} \mathrm{K}$ ). But, when gas and dust start to collapse in a region within the molecular cloud, it slowly heats up. This is a consequence of a law of physics, which tells us that, when matter is squeezed together, the density of the matter will increase and the matter will start to heat up. The outer edge of a collapsing region will have a temperature of around $10^{\circ}$ above absolute zero (also called $10^{\circ} \mathrm{K}$ ), and the inner region will slowly heat up to around $300^{\circ} \mathrm{K}$, which is around room temperature. 
Figure 1

The process of star formation. The illustration shows the six steps of star formation for Sun-like stars. The process starts on (A), where gas and dust in the space between stars (also called the interstellar medium, ISM) collapse into a dense ball of gas called a prestellar core (B) that eventually will become the sun. During the collapse a disk (C) forms around the core, while two jets are emitted at the poles. At some point the star stops growing, but gas still fall onto the disk (D). After a few million years this process also halts. The star is now born (E), while the planets are being formed from the left-over material, which will eventually become a solar system (F). A solar system typically lives 10 billion years after the formation process.

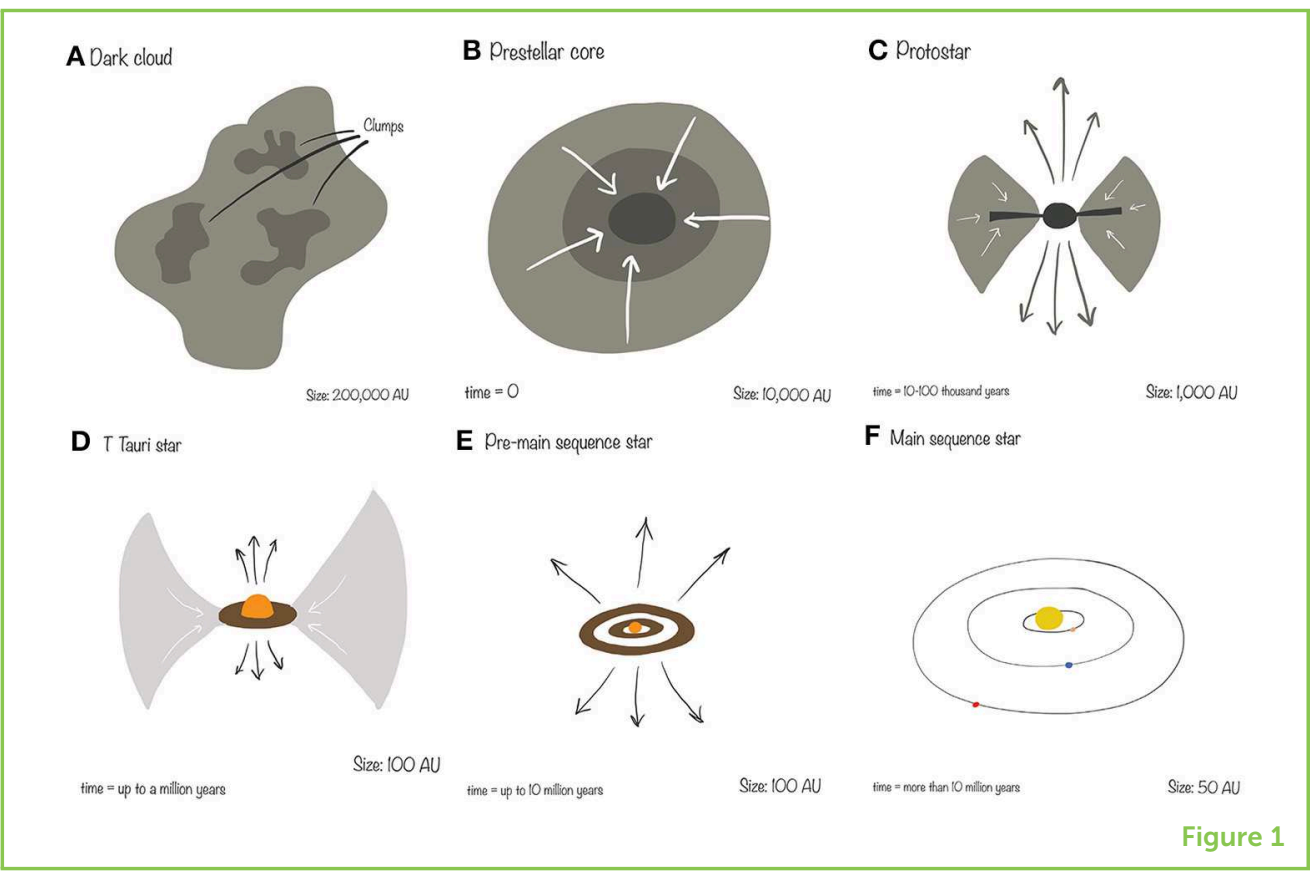

When the collapsing region has reached a size of nearly 10,000 AU, it is called a pre-stellar core (Figure 1B) and is officially a star in-the-making. "Stellar" means star, so pre-stellar means, "before becoming a star." The word core refers to the gas and dust, which are now so dense that the term core is more precise than region or cloud. Also, this pre-stellar core will later become the interior core of the star.

Over the next 50,000 years or so, the pre-stellar core contracts. This might sound like a long time, but on an astronomical timescale it is considered a fairly swift process compared, for instance, to the age of the Universe, which is almost 14 billion years. The core contracts until it is around 1,000 AU (Figure 1C). It is still composed of the same gas and dust, so this means the density of that matter is increasing as the diameter shrinks to 1/10th of the original size of the collapsing region.

After 50,000 years has passed, the system will have formed a disk around the central core, and excess material will be ejected outward from the poles of the star. A pole on a star is like those on the Earth, namely defined as the axis that the star spins around. In Figure 1C, you can see two fountain-like structures where this excess material is ejected. These structures are called jets, and they obey the laws of physics. The random motion of the gas and dust that we described earlier, combined with the system's contraction as the pre-stellar core forms, will cause the whole system to rotate. This process causes a flat disk to form around the pre-stellar core. This is similar to the way a dress forms a flat disk around a spinning ice-skater. If the skater was not rotating, the dress would not be a flat disk around her, but instead would hang along her sides. The jets at the poles arise to keep the 


\section{ACCRETION}

The process where one object accumulates the mass from another object. system in balance. The system is now called a proto-star, which means it is at its very first stage of becoming a real star.

\section{FROM PRE-STELLAR CORE TO STAR}

The disk is crucial for the proto-star to grow into a properly sized star. The disk is mainly composed of gas, which rotates with the disk and slowly approaches the surface of the proto-star. When the gas comes close enough to the star, it falls onto the surface of the star because of gravity, and the star grows. This process of growing is called an accretion process and the star is said to accrete (accumulate) matter from the disk.

Over the next 1,000 years, the matter from the disk is either accreted by the star or expelled from the disk (Figure 1D). The star has grown enough in size and density for the central region to initiate a nuclear reaction, which causes the star to shine, like the Sun. At this point, the star is called a T-tauri star, and this is the first time that the star can be observed visually.

The star eventually stops accreting matter from the disk, but the remaining material around the star is still in a disk-like shape (Figure 1E). The disk no longer serves the purpose of feeding the star with matter to make the star grow. Instead, the disk is now just a circular moving plane of material, which will slowly start to clump together and orbit the star. These small clumps, made from the left-over material from the star's creation, will form new planets. This means that the planets in our solar system are made of the leftover material from the Sun's birth! This is also why all the planets in the solar system are found in the same plane!

The final solar system (Figure 1F) is finished when the disk is completely exhausted, and all the planets are formed. Over the next 10 billion years, the star will burn nuclear fuel in its center and emit energy as the radiation we call sunlight.

\section{OBSERVING MOLECULAR CLOUDS}

Molecular clouds that host and shape newborn stars are dark areas in the night sky. It is not possible for a human to see a molecular cloud-not even with a telescope. The reason that we cannot see a molecular cloud is that dust particles are scattered all over the cloud, and they absorb the light from surrounding stars. This prevents starlight from traveling through space and reaching us here on Earth, which is why a molecular cloud looks like a dark area in the sky. Fortunately for astronomers, the molecular cloud is transparent to radio waves. This means that radio waves are not absorbed by the dust particles in the cloud and the radio waves can therefore travel freely down to us on earth. Radio waves are not visible to the eye, but, using 
big radio telescopes, it is possible to get signals from these otherwise dark molecular clouds.

These radio waves carry information about the contents of the dark molecular cloud. If a star is being born, the cloud will send out different radio waves than if no star is being born within it. The use of radio waves allows astronomers to see when stars are being born, even though the molecular cloud is dark. And, even though astronomers today know a lot about how stars like the Sun are formed, there is actually still a great mystery to be solved.

\section{CONCLUSION}

The great mystery arises when very large stars form. Astronomers know that stars up to about six times the mass of our Sun are born the way we have described in this article. Stars with greater masses require a different process, because the pressure from the stars' radiation will push the disk away, preventing stars from growing larger than roughly six times the size of our Sun.

Astronomers have seen these large stars, so they know large stars exist and must be born in some way. But, how they are born is still a big question for astronomers all over the world.

SUBMITTED: 11 February 2019; ACCEPTED: 19 June 2019;

PUBLISHED ONLINE: 04 July 2019.

EDITED BY: Edward Gomez, Las Cumbres Observatory Global Telescope Network, United States

CITATION: Christensen MBE (2019) How Do Stars Form? Front. Young Minds 7:92. doi: 10.3389/frym.2019.00092

CONFLICT OF INTEREST STATEMENT: The author declares that the research was conducted in the absence of any commercial or financial relationships that could be construed as a potential conflict of interest.

COPYRIGHT @ 2019 Christensen. This is an open-access article distributed under the terms of the Creative Commons Attribution License (CC BY). The use, distribution or reproduction in other forums is permitted, provided the original author(s) and the copyright owner(s) are credited and that the original publication in this journal is cited, in accordance with accepted academic practice. No use, distribution or reproduction is permitted which does not comply with these terms.

\section{YOUNG REVIEWER}

\section{LUKE, AGE: 15}

Hi I am Luke, I am 15, and super interested in astronomy. I also love to read so, when combining the two, you get love to read about astronomy. I like learning about things that I like so I will pay close attention to anything astronomy related. 


\section{AUTHOR}

\section{MAJKEN BRAHE ELLEGAARD CHRISTENSEN}

Majken Christensen is a Danish Astronomer and Science Communicator. She spends her time conveying astronomy to people outside the university walls, and additionally she helps current Astronomy Professors getting their research out in public. Majken has her own company called Astronomicca, where she also runs a blog about astronomy. Previously she has worked at University of Southern Denmark, and before that she has even worked in two different banks, where she did "money-calculations" which were almost as complicated as the calculations of the Universe. *majken.e.c@gmail.com 